\title{
Gasos Guínicos \\ Queratosis Pilar simil asociado a uso de Nilotinib
}

Constanza Ramírez ${ }^{1}$, Sebastián González², Sussan Cuevas²

\section{RESUMEN}

Nilotinib es un inhibidor altamente selectivo de BCR-ABL tirosina kinasa usado para el tratamiento de Leucemia mieloide crónica. Las reacciones cutáneas fueron uno de los efectos adversos no hematológicos más frecuentemente reportados en relación al uso de esta droga.

El presente artículo documenta el caso una paciente femenina de 17 años de edad diagnosticada con Leucemia mieloide crónica que había estado en tratamiento con Nilotinib por 5 meses desarrollando una reacción tipo queratosis pilar. La paciente fue tratada con medidas generales, Urea 15\% y antihistamínicos, con cese del prurito.

Es importante reconocer las reacciones cutáneas asociadas al uso de Nilotinib para así otorgar alivio oportuno de los síntomas con el fin de lograr una mejor adherencia al tratamiento de la Leucemia mieloide crónica y mejorar la calidad de vida del paciente.

Palabras claves: Nilotinib; queratosis pilar; efecto adverso; piel; leucemia mieloide crónica.

\section{SUMMARY}

Nilotinib is a highly selective inhibitor of BCR-ABL tyrosine kinase. It is used as a treatment for chronic myelogenous leukemia (CML). Cutaneous reactions are one of the most common non-hematologic reported adverse effects. The present article documents the case of a 17-year-old female patient diagnosed with CML. She was treated with nilotinib for 5 months and developed a keratosis pilaris-like reaction. The patient was treated with general measures, topical 15\%-urea and antihistamines with improvement and cessation of pruritus.

It is imperative to recognize the cutaneous adverse effects associated with the use of new oncologic treatments such as nilotinib.

Key words: Nilotinib; Keratosis pilaris; Adverse effect; Skin; Chronic myeloid leukaemia.
La leucemia mieloide crónica es un trastorno mieloproliferativo clonal de la médula ósea que se caracteriza por la sobreproducción de células mieloides, fundamentalmente granulocitos maduros. La leucemia mieloide crónica casi siempre presenta cromosoma Philadelphia y/o una alteración del gen BCR-ABL, lo cual produce una proteína con actividad tirosina quinasa. ${ }^{1}$

Este conocimiento ha permitido hoy en día disponer de un tratamiento médico eficaz para esta afectación hema-

${ }^{1}$ Dermatóloga, Hospital Regional de Rancagua, Chile. ${ }^{2}$ Internos de Medicina, Universidad San Sebastián

Correspondencia: Sebastián González Vargas Correo electrónico: sebas13_10@hotmail.com Celular: +5699289 8188. Dirección: Los Claveles 405 casa \#10, San Pedro de la Paz, Concepción, Chile. Código Postal: 4133661 tológica. El manejo de esta patología se realiza principalmente con preparados conocidos como inhibidores de tirosina kinasa, fármacos encargados de bloquear selectivamente la proteína quimérica BCR-ABL, la cual tiene relación directa con la patogenia de la enfermedad. Dentro de estos fármacos encontramos el imatinib, considerado como primera línea de tratamiento. Actualmente, también se cuenta con nilotinib y dasatinib, ambos considerados de segunda generación y usados en casos resistentes o intolerantes a imatinib. En este sentido 
estos fármacos están asociados a múltiples reacciones adversas, destacando entre estas las cutáneas, donde pasa a ser relevante la identificación y manejo adecuado de las mismas, a fin de mejorar tanto la adherencia como el pronóstico de la enfermedad en tratamiento.

Revisaremos a continuación caso clínico que detalla presencia de reacción tipo queratosis pilar secundaria al uso de nilotinib.

\section{Gaso Guínico}

Paciente femenino de 17 años, con antecedentes de leucemia mieloide crónica cariotipo t $(9,22)$ en tratamiento con nilotinib en dosis de $400 \mathrm{mg}$, cada 12 horas desde hace 5 meses, sin antecedentes de atopia ni otras patologías. Consulta por erupción facial pruriginosa, caracterizada por múltiples pápulas foliculares con escamas hiperqueratósicas confluentes en párpados, mejillas, frente, tronco anterior y extremidades superiores, lesiones que iniciaron a los 2 meses de uso del medicamento (Figuras 1 y 2). Debido a los hallazgos clínicos y concordancia temporal se diagnostica una reacción tipo queratosis pilar asociada a nilotinib. Se maneja con preparación magistral Urea 15\%, Ceramidas 3\% en crema base, e Hidroxicina $20 \mathrm{mg}$ en noches. Paciente evoluciona con buena respuesta clínica al presentar cese del prurito, aunque con persistencia de dermatosis, lo que, asociado a estabilidad de cuadro hematológico, permitió continuar el tratamiento con nilotinib.

\section{Discusión}

Nilotinib es un inhibidor de tirosina kinasa de segunda generación utilizado en el tratamiento de tumores con translocación BCR-ABL. Fue aprobado por la FDA el año 2007 para el tratamiento de pacientes con leucemia mieloide crónica Philadelphia (+), resistentes o intolerantes a imatinib. ${ }^{2}$ Estructuralmente el nilotinib es similar al imatinib, pero presenta una potencia y selectividad 30 veces mayor.

En ensayo clínico "ENESTnd" se comprueba su mayor eficacia y seguridad frente al imatinib, siendo así en 2010 aprobado como tratamiento de primera línea para LMC en fase crónica. ${ }^{3}$

A pesar de que los inhibidores de tirosina kinasa son administrados por vía oral y son bien tolerados, su activi-

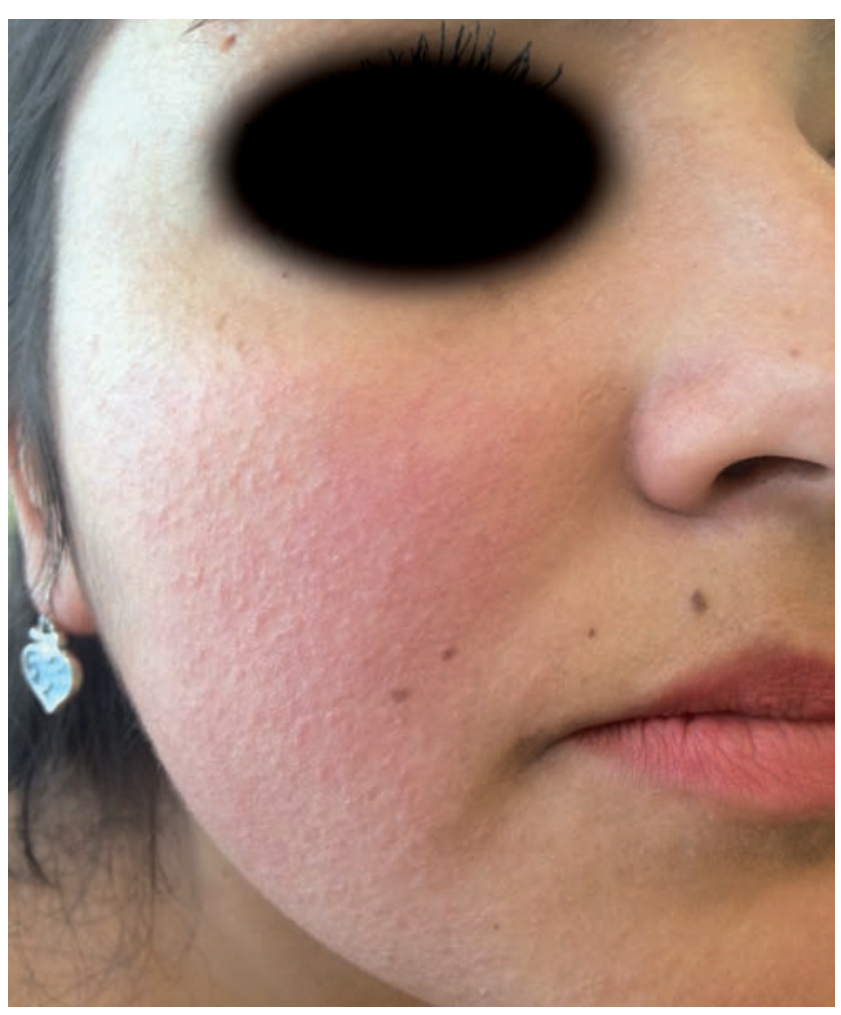

Figura 1 y 2

Pápulas foliculares confluentes.

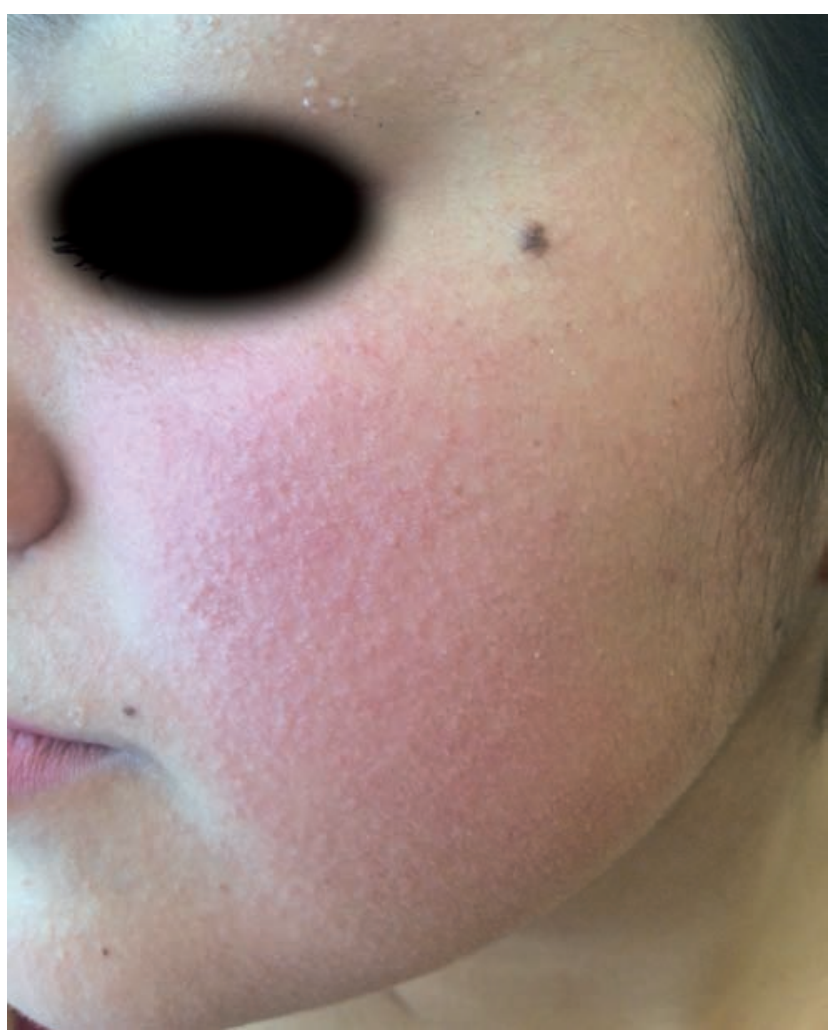


dad no sólo se limita a las células tumorales. Esto puede llegar a ocasionar diversos efectos adversos, encontrándose los cutáneos entre los más frecuentes. De manera que se ha reportado su frecuencia en un $34,3 \%$ de los pacientes que lo utilizan. ${ }^{4}$

Las reacciones adversas cutáneas asociadas a inhibidores de tirosina kinasa de segunda generación, si bien son habituales, tienden a ser menos severas y menos frecuentes que las causadas por imatinib.

A su vez, se ha descrito un $71 \%$ de reacciones cutáneas adversas en pacientes en tratamiento con dasatinib y nilotinib. Dentro de ellas destacan: queratosis pilar extensa $(24 \%)$, erupción maculopapular $(15 \%)$, xerosis $(56,4 \%)$, edema $(33,3 \%)$, alopecia $(41 \%)$ y prurito $(35,9 \%){ }^{5}$

Por lo general la mayoría de estas reacciones son poco sintomáticas y son bien toleradas por los pacientes, por lo que su presencia sumado a un manejo adecuado, no conduce a la suspensión del fármaco, permitiendo la mantención del tratamiento de base y la mejora de la calidad de vida de los pacientes afectados. ${ }^{6}$

Una de las teorías que explica las reacciones cutáneas de los inhibidores de tirosina kinasa se ha relacionado con la actividad cruzada sobre el receptor del factor de crecimiento epidérmico. Mientras que otro mecanismo planteado para explicar la queratosis pilar sería la activación paradójica de la vía MAPK/ERK5.

El hecho de que pacientes presenten manifestaciones como alopecia, queratosis pilar y perdida de vello corporal, hace pensar en un proceso central a nivel folicular, lo cual no ha sido descrito previamente en relación al imatinib. $^{7}$

A pesar de esto, no está del todo clara la fisiopatología de las reacciones adversas cutáneas desencadenadas por los inhibidores de tirosina kinasa de segunda generación.

\section{Conclusión}

Las reacciones tipo queratosis pilar asociadas al uso de nilotinib son raras y de reciente documentación. Si bien no constituye una reacción cutánea severa, puede afectar la calidad de vida de los pacientes que utilizan nilotinib. Consideramos importante el control dermatológico de estos pacientes en vista del uso creciente de estos fármacos y la alta frecuencia de reacciones cutáneas adversas que se han reportado, con el objetivo de educar y advertir sobre la posible aparición de estas mismas, como también otorgar un alivio sintomático oportuno que permita optimizar la adherencia del paciente al tratamiento de su enfermedad de base, mejorando así su calidad y expectativa de vida.

\section{REFERENCIAS}

1. González V, Gutiérrez F, Gavira R, Viña M, Moreno M, Gázquez $\mathrm{R}$. Adherencia y toxicidad de los inhibidores de la tirosinquinasa en leucemia mieloide crónica. Farm Hosp. 2013;37(6):434-440

2. Kantarjian HM, Hochhaus A, Saglio G, De Souza C, Flinn IW, Stenke L, et al. Nilotinib versus imatinib for the treatment of patients with newly diagnosed chronic phase, Philadelphia chromosomepositive, chronic myeloid leukaemia: 24-month minimum follow-up of the phase 3 randomised ENESTnd trial. Lancet Oncol. 2011 Sep;12(9):841-51

3. Hochhaus A, Saglio G, Hughes T, Larson R, Kim D, Issaragrisil S, et al. Long-term benefits and risks of frontline nilotinib vs imatinib for chronic myeloid leukemia in chronic phase: 5-year update of the randomized ENESTnd trial. Leukemia 2016 (30),1044-1054. Disponible en: www.nature.com (Consultado el 3 de marzo de 2017)

4. Leong W, Aw G. Nilotinib-Induced Keratosis Pilaris. Case Rep Dermatol 2016;8(1):91-96

5. Delgado L, Giraudier S, Ortonne N, Zebou O, Cordonnier C, Hulin $\mathrm{A}$, et al. Adverse cutaneous reactions to the new second-generation tyrosine kinase inhibitors (dasatinib, nilotinib) in chronic myeloid leukemia. J Am Acad Dermatol. 2013;69(5):839-840

6. García-Zamora E, Naz E, Floristán U, López-EstebaránzJ. Erupción tipo queratosis pilar asociada a nilotinib. Piel 2017;32(4):244-247

7. Patel A, Solomon A, Mauro M, Ehst B. Unique Cutaneous Reaction to Second- and Third-Generation Tyrosine Kinase Inhibitors for Chronic Myeloid Leukemia. Dermatology 2016, 232(1):122-125 